\title{
Estrogen and high-fat diet induced alterations in C57BL/6 mice endometrial transcriptome profile
}

\author{
Yali Cheng ${ }^{1, *}$, Qiaoying Lv1,*, Bingying Xie', Bingyi Yang ${ }^{1}$, Weiwei Shan ${ }^{1}$, Chengcheng Ning ${ }^{1}$, Bing Li $^{1}$, \\ Liying Xie', Chao Gu' ${ }^{1}$, Xuezhen Luo' ${ }^{1}$, Xiaojun Chen ${ }^{1}$ and Qin Zhu \\ 1Department of Gynecology, Obstetrics and Gynecology Hospital of Fudan University, Shanghai, China \\ ${ }^{2}$ Department of Pathology, Obstetrics and Gynecology, Hospital of Fudan University, Shanghai, China
}

Correspondence should be addressed to X Chen or Q Zhu: xiaojunchen2013@sina.com or zhuq1215@163.com

*(Y Cheng and Q Lv contributed equally to this work)

\begin{abstract}
Unopposed estrogen stimulation and insulin resistance are known to play important roles in endometrial cancer (EC), but the interaction between these two factors and how they contribute to endometrial lesions are not completely elucidated. To investigate the endometrial transcriptome profile and the associated molecular pathway alterations, we established an ovariectomized C57BL/6 mouse model treated with subcutaneous implantation of 17- $\beta$ estradiol (E2) pellet and/or high-fat diet (HFD) for 12 weeks to mimic sustained estrogen stimulation and insulin resistance. Histomorphologically, we found that both E2 and E2 + HFD groups showed markedly enlarged uterus and increased number of endometrial glands. The endometrium samples were collected for microarray assay. GO and KEGG analysis showed that genes regulated by E2 and/or HFD are mainly responsible for immune response, inflammatory response and metabolic pathways. Further IPA analysis demonstrated that the acute phase response signaling, NF- $\kappa B$ signaling, leukocyte extravasation signaling, PPAR signaling and LXR/RXR activation pathways are mainly involved in the pathways above. In addition, the genes modulated reciprocally by E2 and/or HFD were also analyzed, and their crosstalk mainly focuses on enhancing one another's activity. The combination analysis of microarray data and TCGA database provided potential diagnostic or therapeutic targets for EC. Further validation was performed in mice endometrium and human EC cell lines. In conclusion, this study unraveled the endometrial transcriptome profile alterations affected by E2 and/or HFD that may disturb endometrial homeostasis and contribute to the development of endometrial hyperplasia.
\end{abstract}

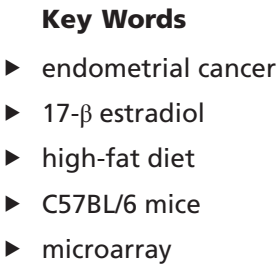

Endocrine Connections (2018) 7, 36-46

\section{Introduction}

Endometrial cancer (EC) is one of the most common malignant genital tumors among females worldwide, with an estimated 61,380 new cases in America in 2017 (1). It is known that type1 EC is estrogen-dependent tumor. Sustained endogenous or exogenous estrogen

http://www.endocrineconnections.org https://doi.org/10.1530/EC-17-0315

(c) 2018 The author Published by Bioscientifica Ltd exposure without progesterone protection is the leading cause of type1 EC $(2,3)$. Accumulating epidemiological evidence also demonstrated that insulin resistance was highly prevalent in endometrial cancer $(4,5,6)$. Our previous study reported that hyperinsulinemia might 
be a key factor that initiates and promotes both type 1 EC and endometrial hyperplastic lesions including disordered proliferative endometrium (DPE) and endometrial hyperplasia (EH) with or without atypia (7). Thus, elucidation of the molecular mechanisms of insulin resistance in estrogen-related EC and potential crosstalk will benefit our understanding of endometrial lesions.

Insulin resistance has been reported to upregulate $17-\beta$ estradiol (E2) biosynthesis and estrogen receptor to increase the estrogen sensitivity in local endometrium. Mechanistically, chronic inflammation induced by insulin resistance might be the key modulator for estrogen activity (8). Studies demonstrated that increased IL6 promotes the production of aromatase by stromal cells, resulting in an increase of E2 biosynthesis $(9,10)$. IL17A, secreted by macrophages in EC patients, upregulates the expression of estrogen receptor alpha (ER $\alpha)$ (11). Furthermore, both insulin and IGF1 intervention could upregulate G protein-coupled estrogen receptor (GPER) expression through epigenetic modulation to increase the sensitivity of endometrial cells to estrogen $(12,13)$. Collectively, insulin resistance, chronic inflammation and estrogen sensitivity are critical to endometrial tumorigenesis.

To investigate the interaction between insulin resistance and estrogen in endometrial carcinogenesis, we established an ovariectomized C57BL/6 mouse model treated with subcutaneous implantation of estrogen pellet and/or high-fat diet to mimic sustained estrogen stimulation and insulin resistance. The present study unraveled alterations in endometrial transcriptome profile and associated molecular pathways, which might disturb the homeostasis of the endometrial environment.

\section{Materials and methods}

\section{Mice}

Forty female C57BL/6 mice were purchased from the Laboratory Animal Science of Shanghai Medical College, Fudan University (Shanghai, China) and used for this study. Mice were maintained in a specific pathogenfree (SPF) and temperature-controlled facility on a 12-h light/darkness cycle. All experimental procedures were conducted in accordance with the National Guidelines for Animal Use in Research (China) and approved by the Institutional Animal Care and Use Committee of Fudan University.

$$
\begin{aligned}
& \text { http://www.endocrineconnections.org } \\
& \text { https://doi.org/10.1530/EC-17-0315 }
\end{aligned}
$$

(c) 2018 The authors Published by Bioscientifica Ltd

\section{Study design}

All mice received bilateral oophorectomy at 6-8 weeks after birth. Successful ovariectomy was confirmed by microscopic examination of a vaginal smear for ten consecutive days. The mice were then randomly divided into four groups including the control (Ctrl) group, the estrogen stimulation (E2) group, the high-fat diet (HFD) group and the estrogen plus high-fat diet (E2 + HFD) group ( $n=8 /$ group). The Ctrl group was treated with a normal chow diet, and the E2 group received a normal chow diet and an E2-releasing pellet (Innovative Research of America, NE-121, 0.36 mg/pellet 90-day release) implanted subcutaneously. The HFD group was fed high-fat diet (Research Diets, D12492) and the E2 + HFD group received both an E2-releasing pellet implanted subcutaneously and high-fat diet. All mice were killed after 12 weeks of treatment. Body weight and uterine morphology were recorded, and the endometrium was collected for microarray assay.

\section{Endometrium collection and mRNA extraction}

Mouse uterus was longitudinally dissected and scraped using the back of a scalpel into RNA Later on ice. Total RNA was extracted using an RNeasy Mini-kit (Qiagen) according to the manufacturer's protocol. Agarose gel electrophoresis assay was used to confirm the presence of total RNA with no degradation.

\section{Microarray analysis}

The total RNA samples isolated from the Ctrl, E2, HFD and $\mathrm{E} 2+\mathrm{HFD}$ groups in three or four biological replicates were subjected to gene expression analysis using the Affymetrix GeneChip Mouse Transcriptome Array 1.0 by GMINIX (Shanghai, China). A stringent significance threshold $(P<0.05)$ and false discovery rate $(\mathrm{FDR}<0.05)$ were used to limit the number of false-positive findings, and the fold change $(>1.5$ or $<0.67$ ) was chosen to screen a manageable number of genes. According to differential expression of these genes, heat map and hierarchical clustering were used based on a complete-linkage hierarchical clustering algorithm.

\section{Function and pathway analysis of genes}

The Gene Ontology (GO) Database was used to search for the most significant function of dysregulated genes from the levels of biological process, cellular component and

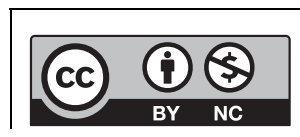

This work is licensed under a Creative Commons Attribution-NonCommercial 4.0 International License. 
molecular function. The Kyoto Encyclopedia of Genes and Genomes (KEGG) database was used to analyze the canonical pathways of dysregulated genes. Fisher's exact test and multiple comparison tests were used for GO and KEGG analysis, with a $P$ value of $<0.05$ and an FDR of $<0.05$ considered statistically significant. Aside from the functional analysis, canonical pathways and networks of differential genes were also analyzed using ingenuity pathways analysis (IPA).

\section{Hematoxylin-eosin (HE) staining and immunohistochemical (IHC) staining}

The HE staining was mainly used to evaluate pathological progress. Three most representative $\times 100$ fields per slide were selected and gland number in glandular epithelium was calculated. We observed the diameter of gland in Ctrl group is about $40-50 \mu \mathrm{m}$, then we calculated the percentage of gland (diameter $>50 \mu \mathrm{m}$ ) in total glands to evaluate the gland size. The IHC assay was performed as described before (14). Primary antibodies to PGR, CCND1, STMN1, PCNA and TNFAIP3 were purchased from Abcam. LGR5, VIM and KRT4 antibodies were purchased from Sigma-Aldrich. Phosphate buffered saline (PBS) was used as a negative control.

\section{Cell lines, cell treatment and quantitative real-time PCR (qRT-PCR)}

Human endometrial cancer cell lines Ishikawa and ECC1 were purchased from ATCC and maintained in DMEM/ F12 (HyClone, Logan, UT, USA) and RPMI-1640 medium (HyClone) containing 10\% charcoal-stripped fetal bovine serum (Gibco) and 100 U/mL penicillin and streptomycin antibiotics separately. Cells were treated with $10^{-7} \mathrm{M}$ E2 (Sigma-Aldrich, E2758) for $12 \mathrm{~h}$ before total RNA was harvested for extraction. QRT-PCR was carried out according to the manufacturer's protocols (SYBR Premix Ex Taq, TaKaRa, China). Primers were synthesized by Sangon Biotech (Shanghai, China), and they are listed in Supplementary Table 1 (see section on supplementary data given at the end of this article).

\section{Availability of supporting data}

The raw and normalized data gene expression data used in this manuscript have been deposited in the Gene Expression Omnibus repository, with GEO accession number GSE102103 (https://www.ncbi.nlm.nih.gov/geo/ query/acc.cgi?acc=GSE102103).
The cancer genome atlas (TCGA) data analysis

Significantly differentially expressed genes were interrogated into the cBioPortal database (http://www. cbioportal.org) for transcriptomic analysis. We queried all the samples from TCGA uterine corpus endometrial carcinoma (UCEC) (TCGA, provisional) with RNAseq v2 data $(n=373)$ in our study and considered RNA dysregulation with $Z$-score threshold: \pm 2 (15). We also showed the expression levels of the dysregulated genes between cancer tissues and para-noncancerous tissues with the assistance of GEPIA (http://gepia.cancer-pku.cn), which could analyze the RNA sequencing expression data of 23 types of cancers and normal samples from the TCGA according to the standard processing pipeline.

\section{Statistical analysis}

SPSS 19.0 (IBM SPSS Software) was used for statistics. Unpaired $t$ test was used for appropriate analyses. The $P$ value of $<0.05$ (two-tailed) was considered to be significant. In 'enhancement' analysis, we analyzed gene expression of 173 overlapped genes among 13 samples from four groups. Fold change was calculated by the value of genes in treatment groups normalized to those in control group. We regarded the fold change $>1.5$ as 'enhanced' and $<0.67$ as 'repressed' as previously described (16).

\section{Results}

\section{Uterine size and histological performance}

To confirm the effect of continuous estrogen exposure and/or high-fat diet on mouse uteri, we first investigated body weight and uterine morphology after 12 weeks of treatment. The HFD and E2 + HFD mice significantly had higher body weight than Ctrl (Fig. 1A). The E2 and E2 + HFD groups showed a markedly enlarged uterine size (hypertrophic uterus), while the HFD group showed slight uterine edema compared with Ctrl group (Fig. 1B). We examined the endometrial histology using $\mathrm{H} \& \mathrm{E}$ staining. Although the endometrium showed a benign proliferative status in all groups, we did find increased endometrial gland numbers in the $\mathrm{E} 2$ group, increased gland size in the HFD group and increased gland numbers of various sizes in the E2+HFD group (Fig. 1C and $\mathrm{D}$ ). These findings indicated that sustained estrogen stimulation and high-fat diet could facilitate uterine hypertrophy and the proliferation of endometrial gland tissue.

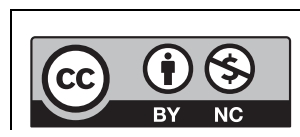

This work is licensed under a Creative Commons Attribution-NonCommercial 4.0 International License. 
B
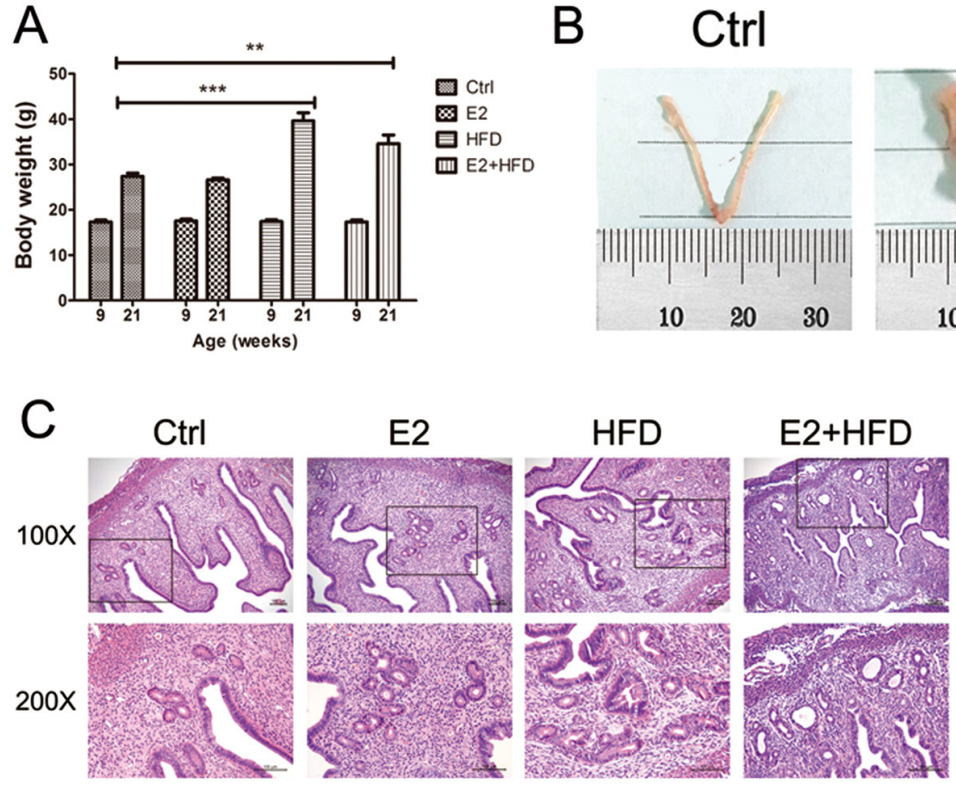

E2

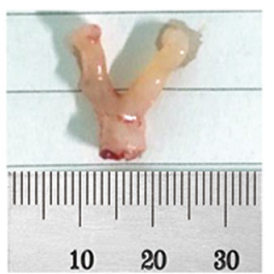

HFD

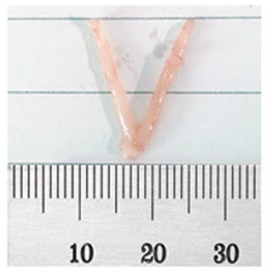

E2+HFD

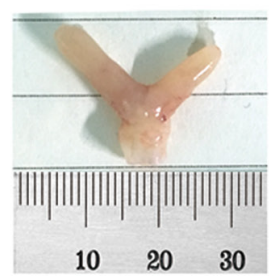

Figure 1

Uterine size and histological performance. (A) Body weight change at the beginning (9 weeks) and end ( 21 weeks) of treatment ( $n=8 /$ group). (B) Representative morphological images of uteri from mice with estrogen and/or high-fat diet treatment. Ctrl, Control; E2, Estrogen; HFD, High-fat Diet. Ruler units, $1 \mathrm{~mm}$. (C) Hematoxylin-eosin (H\&E) staining of endometrium from mice with estrogen and/or high-fat diet treatment. The boxed areas in the top panel $(100 x)$ are magnified in the bottom panel $(200 x)$. Scale bars, $100 \mu \mathrm{m}$. (D) Gland number and size evaluation. The left panel represents the change of gland number per field, and the right panel represents the percentage of gland (diameter $>50 \mu \mathrm{m}$ ) in total glands per field. * $P<0.05$, $* * P<0.01, * * * P<0.001$, n.s, no significant.

\section{Endometrial mRNA profile in mice treated with estrogen and/or high-fat diet}

To investigate the endometrial transcriptome profile induced by continuous estrogen exposure and/or high-fat diet in ovariectomized mice, we collected the endometrium for microarray analysis. Our primary microarray data are available in the public database Gene Expression Omnibus (GEO) repository (GSE102103). When hierarchical clustering was applied to the microarray data, samples with similar trends in gene expression profiles tend to cluster together. The mRNA expression profiles were presented in Fig. 2A. The number of differentially expressed genes regulated by E2 and/or HFD was shown in Fig. 2B. It is noteworthy that the E2 + HFD group showed the highest number of dysregulated genes compared to E2 and HFD groups.

To gain insight into the functional alterations of these dysregulated genes, ontology enrichment analysis was performed. Among the altered functions, 90 GO terms were all enriched in the E2 and/or the HFD groups compared to the Ctrl group. Focusing on the top ten terms of the three treatment groups, we found that the inflammatory response, the innate immune response and cell adhesion were affected (Fig. 2C). We further used KEGG to analyze the signaling pathways in which these aberrant genes are involved. Among the altered pathways, 43 pathways were all enriched in the three groups when compared to Ctrl group. Concentrating on the top ten KEGG pathways of each group, we observed that cytokinecytokine receptor interaction, Staphylococcus aureus infection and the phagosome pathway were commonly enriched (Fig. 2D). Notably, among the GO and KEGG analysis, metabolic-related functions and pathways were significantly enriched in HFD and E2 + HFD groups. Taken together, these results suggest that estrogen and high-fat diet may regulate the immune response, the inflammatory response and the metabolic microenvironment to induce endometrial disorders.

\section{Estrogen and high-fat diet synergistically promote endometrial lesions}

As clinical research has demonstrated that prolonged estrogen stimulation and insulin resistance are contributing factors for endometrial lesions, we supposed that estrogen and insulin resistance might have synergic effects on endometrial carcinogenesis. First, we screened 173 common genes (148 upregulated genes and 25 downregulated genes) from E2 and/or HFD groups compared to the Ctrl group (Fig. 3A). The expression heat http://www.endocrineconnections.org https://doi.org/10.1530/EC-17-0315 (c) 2018 The authors Published by Bioscientifica Ltd

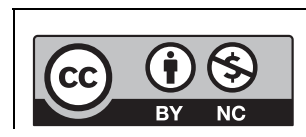

This work is licensed under a Creative Commons Attribution-NonCommercial 4.0 International License. 
A

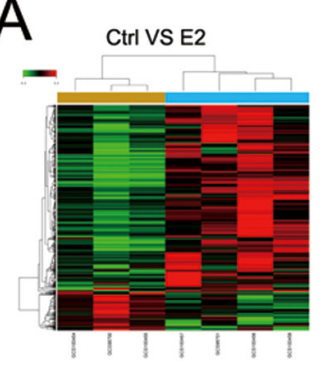

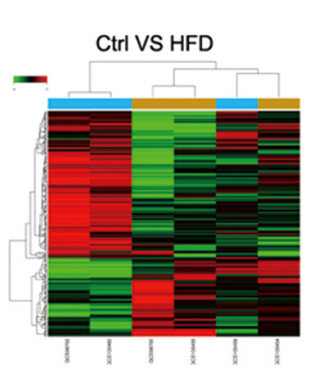

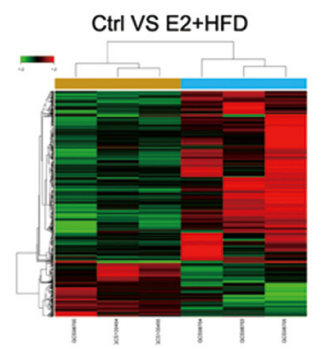

B

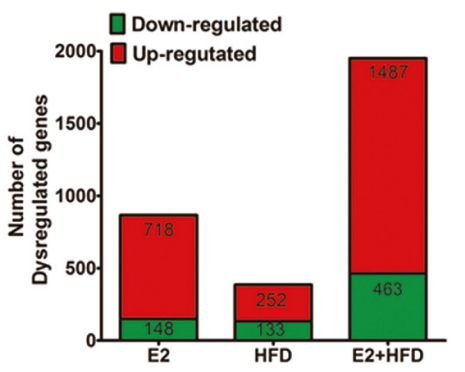

C

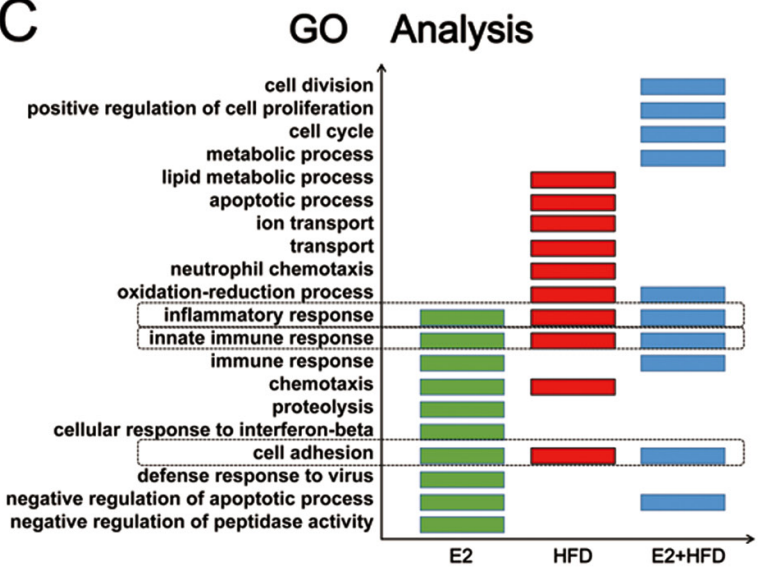

D

\section{KEGG Analysis}

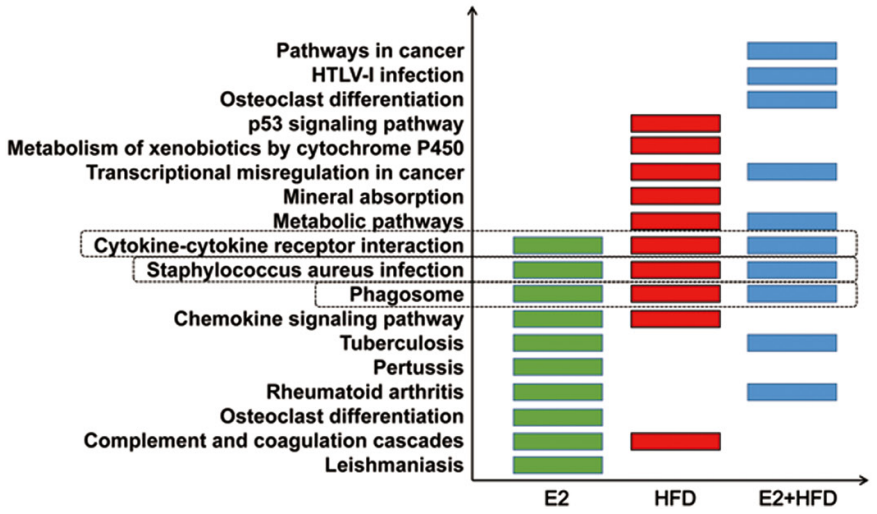

Figure 2

Changes of mRNA profiles in mice treated with estrogen and/or high-fat diet. (A) Cluster analysis of dysregulated gene expression in the endometrium of mice. Samples in the heat map are labeled: GCS (100454, 100455, 98700) for Ctrl, GCS $(100456-100458,98701)$ for E2, GCS (100459, 100460, 98702) for HFD and GCS (98703-98705) for E2 + HFD. Red color represents highly expressed genes and green color represents lowly expressed genes. (B) The number of altered genes regulated by E2, HFD and E2 + HFD. The number of genes upregulated or downregulated (with $>1.5-$ or $<0.67-$ fold change compared to Ctrl group) are represented as red and green numbers, respectively. (C) GO analysis of E2, HFD and E2 + HFD regulated genes in mouse endometrial samples. The top ten functions are presented and overlapped functions are boxed. (D) KEGG analysis of E2, HFD and E2 + HFD regulated genes in mouse endometrial samples. The top ten pathways are presented and overlapped pathways are boxed.

map of these genes is shown in Fig. 3B. To investigate the collaborative effect of E2 and HFD, we compared the effects of E2+HFD and E2/HFD among the 173 genes. We found that HFD enhances E2 activity for approximately $33 \%$, while it reverses or represses E2 action on 3\% of these genes (Fig. 3C). Similarly, E2 enhanced HFD activity up to approximately $54 \%$ of these genes but reversed or repressed HFD action on only $2 \%$ of these genes (Fig. 3C). Enhanced and reversed genes controlled reciprocally by E2 and HFD are listed in Supplementary Table 2. In conclusion, E2 and HFD modulate gene activity reciprocally by enhancing each other's activity.

To explore the potential synergic mechanisms, we conducted ingenuity pathways analysis (IPA) for these 173 genes. The enriched top ten pathways were shown in Table 1. Granulocyte adhesion and diapedesis, acute phase response signaling and NF- $\mathrm{kB}$ signaling were associated with inflammatory responses; LXR/RXR activation, PPAR signaling and the urea cycle were related to metabolic pathways; and the complement system and communication between innate and adaptive immune cells were associated with immune responses. IPA analysis results were also consistent with GO and KEGG analysis (Fig. 2C and D). Among these pathways, acute phase response signaling, dendritic cell maturation and NF- $\kappa$ B signaling were activated, while PPAR signaling and the LXR/RXR activation pathway were inhibited. These results further confirmed that estrogen and high-fat diet might synergistically regulate the immune response, as well as inflammatory and metabolic pathways. These results also indicated that the PPAR signaling pathway and pathways involved in communication between innate and adaptive immune cell might represent future research directions to investigate the mechanism of sustained estrogen and high-fat diet-induced endometrial lesions.

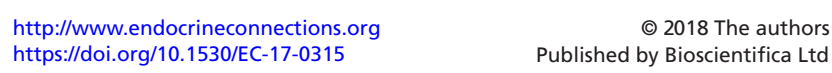



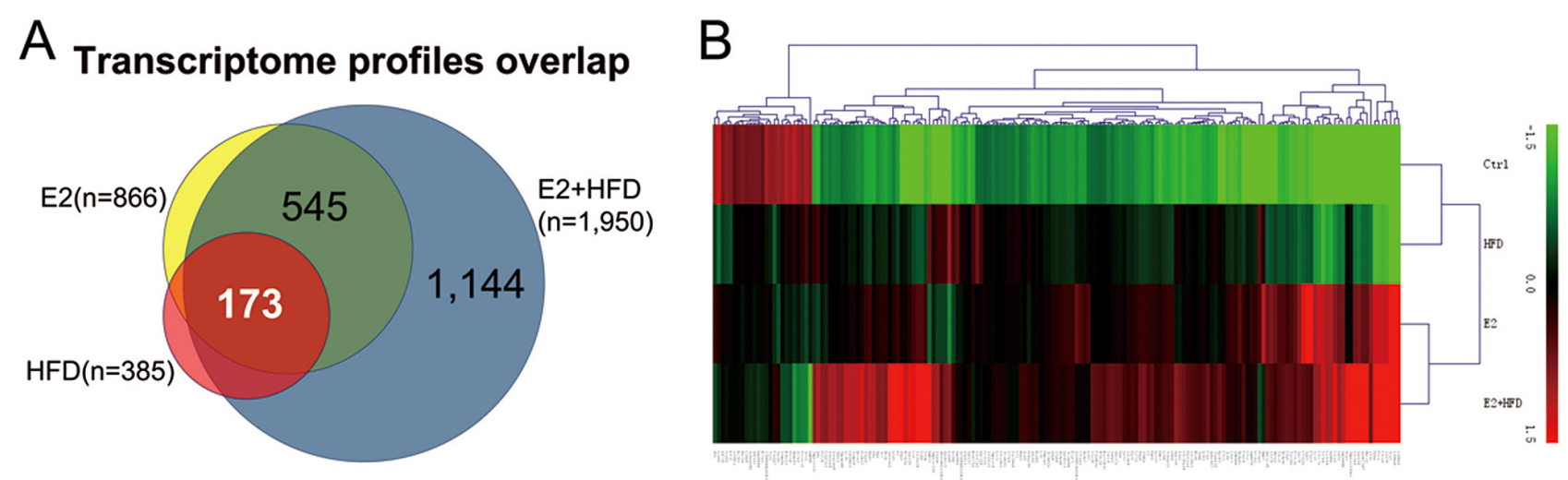

C Overlapped genes $(\mathrm{N}=173)$

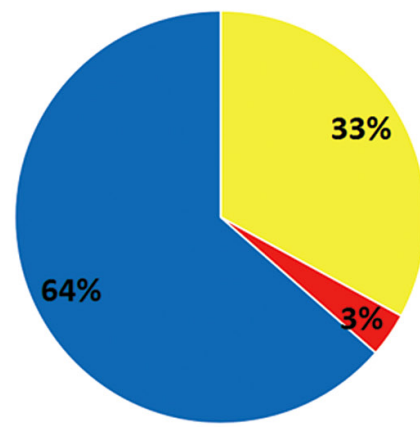

HFD enhances E2 activity - HFD reverses E2 activity - HFD has no effect on E2 activity

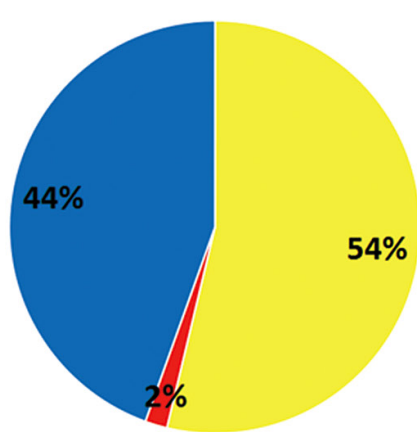

E2 enhances HFD activity

- E2 reverses HFD activity

- E2 has no effect on

HFD activity

Figure 3

Estrogen and high-fat diet synergically promote endometrial lesions. (A) The 173 commonly upregulated and downregulated genes of mouse endometrial microarray in E2, HFD and E2 + HFD groups compared to Ctrl group. (B) Heat map illustrating the expression patterns of the 173 commonly dysregulated genes in E2 and/or HFD. The red and green colors indicate high and low expression level of genes, respectively. (C) E2 and HFD control gene modulation reciprocally. The left pie chart is the effect of HFD on common differentially expressed genes in mouse endometrium. The right pie chart is the effect of E2 on common differential genes in mice endometrium. We regarded the fold change $>1.5$ as 'enhanced' and $<0.67$ as 'repressed'.

Table 1 Functional annotations enriched by the differential expressed genes, detected by using the IPA library of canonical pathways.

\begin{tabular}{l} 
Ingenuity canonical pathways \\
\hline Granulocyte adhesion and diapedesis \\
Complement system \\
LXR/RXR activation \\
Communication between innate and \\
adaptive immune cells \\
Acute phase response signaling \\
Phagosome formation \\
Urea cycle \\
Leukocyte extravasation signaling \\
PPAR signaling \\
NF- $\mathrm{KB}$ signaling
\end{tabular}

\begin{tabular}{|c|c|}
\hline$P$ value & Z-score \\
\hline $4.36516 \mathrm{E}-08$ & - \\
\hline 1.94984E-07 & - \\
\hline 2.13796E-05 & -1.134 \\
\hline 3.54813E-05 & \\
\hline 0.000177828 & 2 \\
\hline 0.00020893 & - \\
\hline 0.000707946 & - \\
\hline 0.003467369 & 1 \\
\hline 0.003981072 & -2 \\
\hline 0.00831763 & 1.342 \\
\hline
\end{tabular}

The top ten pathways are presented. $Z$-score $>0$ indicates activation and $Z$-score $<0$ indicates inhibition.

\section{Aberrantly expressed genes analysis based on TCGA data}

TCGA database has significantly improved the understanding of UCEC (15). According to the degree of aberration in 173 dysregulated genes in patients, we listed the OncoPrint from cBioPortal involving the top fifteen genes with their respective abnormal expression profiles. Genetic alterations were found in 225 of 333 UCEC patients (68\%). The aberrant expression threshold was defined as $Z$-score \pm 2.0 from the TCGA RNA-Seq V2 data (Fig. 4A). Then, we analyzed the expression of those genes in EC and para-tumorous endometrial tissues. The results indicated that the levels of PIGR, KCTD1, ARG2, PMAIP1, ALPL and SLC27A2 were remarkably higher in the UCEC than in the para-tumorous uterine tissues. In contrast, FMO2 and CCND2 were all downregulated in UCEC tissues (Fig. 4B). A combination of microarray 
A

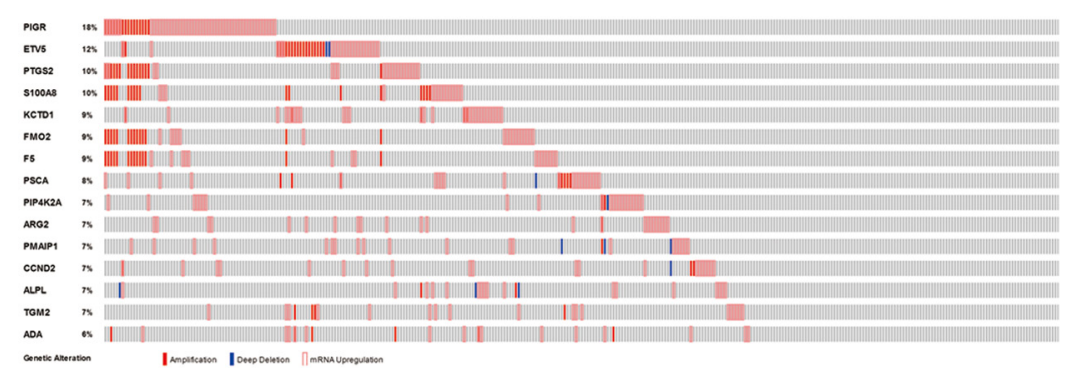

Figure 4

Aberrantly expressed genes analysis based on TCGA data. (A) Genetic alterations. Red represents amplification, blue represents deep deletion and pink represents mRNA upregulation. Genetic alterations were found in 225 of 333 UCEC patients (68\%). The aberrant expression threshold was defined as $Z$-score \pm 2.0 from the TCGA RNA-Seq V2 data. (B) Comparisons of genes (PIGR, KCTD1, FMO2, ARG2, PMAIP1, CCND2, ALPL, SLC27A2) expression between UCEC cancer

$\mathrm{B}$
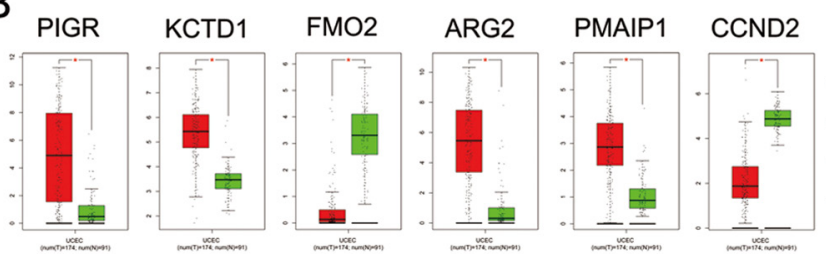

and TCGA data provided potential diagnostic molecular markers for high-fat diet and estrogen-induced endometrial lesions.

\section{Microarray validation in mouse endometrial samples and EC cell lines}

To verify the accuracy and reliability of the microarray results, we first used quantitative real-time PCR (qRTPCR) and immunohistochemistry (IHC) staining in mouse endometrium to detect gene transcription and protein expression, respectively. QRT-PCR validated the transcription level of eight differentially genes (Tnfaip3, Neurl3, Ihh, Plk1, Ccnf, Peli3, Mdm2 and Skp2) in mouse endometrium of the E2 group (Fig. 5A). Seven out of eight genes were consistent with the microarray results. IHC staining showed decreased expression of PGR, CCND1, PCNA, STMN1 and LGR5, while showing increased expression of TNFAIP3, VIM and KRT4 in the E2 and/or the HFD group compared with Ctrl group (Fig. 5B and C). These IHC results are also consistent with the microarray data. Furthermore, estrogentreated human endometrial cancer cell lines (Ishikawa and ECC1) were used to detect gene transcription level (Fig. 5D). We also investigated the effect of high-fat diet-induced insulin resistance on endometrium by analyzing mRNA level of genes accompanied by insulin resistance and inhibited insulin signaling pathway in HFD group. Data were shown in Supplementary Table 3. These results not only indicated the achievement of insulin resistance indirectly, but also proved that the microarray data in mouse endometrial samples are accurate and reliable.

http://www.endocrineconnections.org https://doi.org/10.1530/EC-17-0315

2018 The authors Published by Bioscientifica Ltd

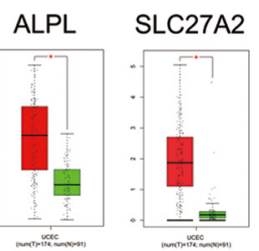
tissues and non-cancerous tissues involved in TCGA based on GEPIA. $Y$ axis indicates the log2 $(T P M+1)$ for genes expression. Red bar shows the tumor tissues and green bar indicates the non-cancerous tissues. These figures were derived from Gene Expression Profiling Interactive Analysis (GEPIA). ${ }^{*} P<0.01$. TPM, Transcripts per Kilobase Million.

\section{Discussion}

Accumulating evidence suggests that insulin resistance, which is the main characteristic of obesity (17), type 2 diabetes mellitus (18) and polycystic ovary syndrome (PCOS) (10), is the risk factor for EC. A prospective cohort study notes that 'insulin resistance/metabolic syndrome,' 'steroids,' and 'inflammation' factors are the three risk components associated with postmenopausal EC (19). Based on the clinical findings, we established a mouse model to find endometrial alterations of transcriptome profile under E2 and/or HFD treatment. Here, we compared the uterus size, endometrial histology and transcriptome profile in E2 and/or HFD mice. Immune response, inflammatory response and metabolic pathways were most affected by both E2 and/or HFD treatment. Furthermore, dysregulated genes enriched in the three groups were analyzed to examine the crosstalk between estrogen and insulin resistance. The functions and pathways of the genes altered in E2 and/or HFD mice endometrium will help discover the mechanisms of endometrial tumorigenesis at the earlier stages.

During the 12 weeks of estrogen treatment in our mouse model, we found both enlarged uterine size and increased endometrial gland number but were not able to diagnose $\mathrm{EH}$. These results partly differ from findings in an estrogen-induced EH mouse model. In that model, female BALB/c mice without ovariectomy were subcutaneously implanted with $\sim 30 \mathrm{mg} 17-\beta$ estradiol for ten weeks, which led to pathologically elevated serum E2 levels (up to 20-fold compared with physiological level) (20). Conversely, in our mouse model, we selected a $0.36 \mathrm{mg}$ $17-\beta$ estradiol pellet released subcutaneously over 90 days.

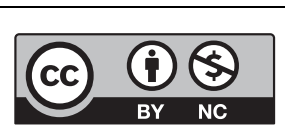

This work is licensed under a Creative Commons Attribution-NonCommercial 4.0 International License. 
A

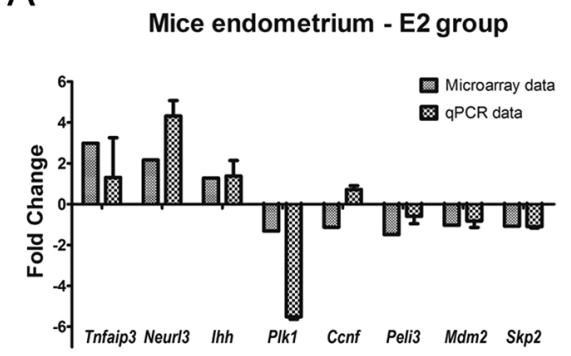

B

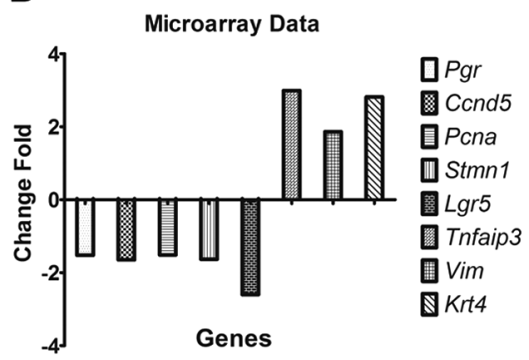

C

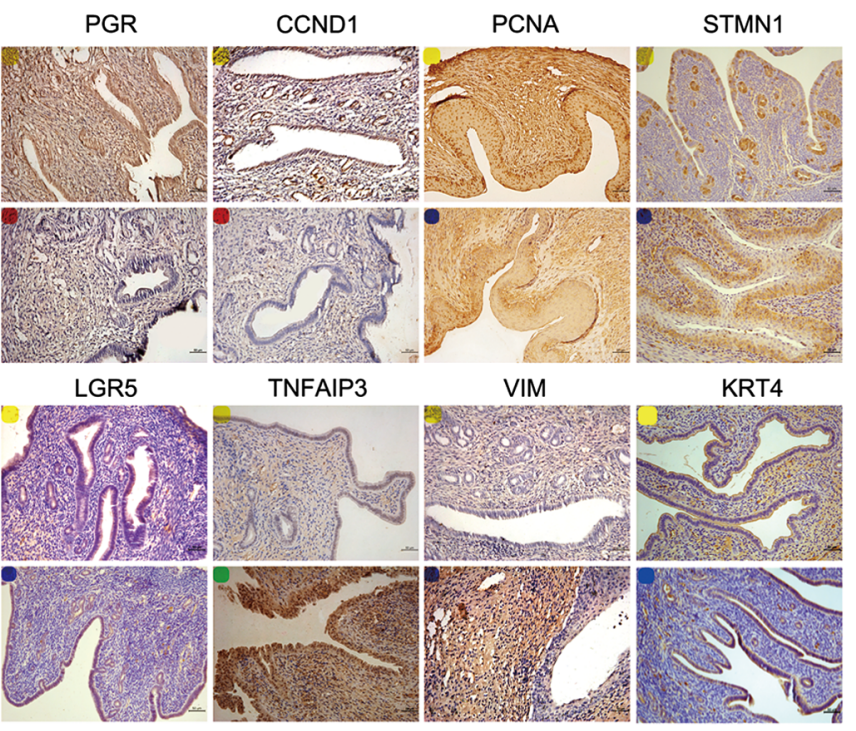

D

Estrogen-treated EC cell lines

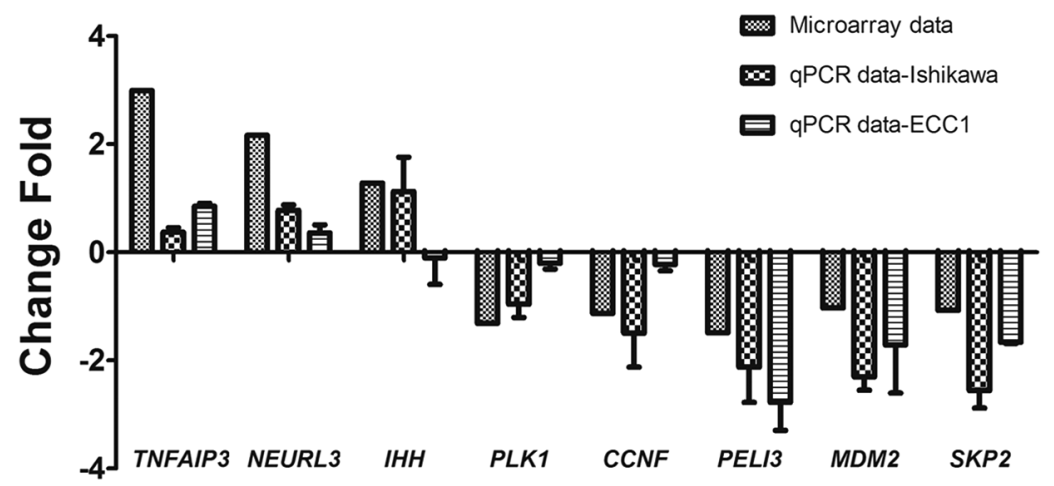

\section{Figure 5}

Microarray validation in mouse endometrial samples and EC cell lines. (A) Microarray validation by qRT-PCR in mice endometrium. The expression levels of genes Tnfaip3, Neurl3, Ihh, Plk1, Ccnf, Peli3, Mdm2 and Skp2 from microarray analysis in comparison with qRT-PCR gene expression levels are presented. These genes were normalized to GAPDH and then calculated as fold changes of relative mRNA expression. (B and C) IHC staining of PGR, CCND1, PCNA, STMN1, LGR5, TNFAIP3, VIM and KRT4 (B) are consistent with the microarray data (C) in E2 and/or HFD group vs Ctrl group. Yellow, Ctrl group; Green, E2 group; Red, HFD group; Blue, E2 + HFD group. Scale bars, $100 \mu \mathrm{m}$. (D) Microarray validation by $\mathrm{qRT}$-PCR in human endometrial cancer cell lines. The expression levels of genes TNFAIP3, NEURL3, IHH, PLK1, CCNF, PELI3, MDM2 and SKP2 from microarray analysis compared to qRT-PCR gene expression levels are presented.

These genes were normalized to GAPDH and calculated as fold changes in relative mRNA expression.
This small amount of estrogen treatment is conducive to understanding the very early changes of endometrial lesions but not sufficient to induce pathological EH.

In our study, high-fat diet for 12 weeks resulted in significant weight gain in female C57BL/6 mice. In addition, transcript levels were decreased for Irs1, G6pc3, $A k t 3$, increased for Igf1, Tnfrsf11a (NF- $\kappa B)$, Insig1, Ppa2 and PPAR signaling pathway appeared to be inhibited. These genetic features are often associated with insulin resistance $(21,22,23)$. And the conclusion that insulin resistance

$$
\begin{array}{lr}
\text { http://www.endocrineconnections.org } & \odot 2018 \text { The authors } \\
\text { https://doi.org/10.1530/EC-17-0315 } & \text { Published by Bioscientifica Ltd }
\end{array}
$$

could be successfully induced by high-fat diet in mice for 12 weeks was supported by several studies. They reported that mice fed with high-fat diet had increased body weight, hyperglycemia, hyperinsulinemia and increased total cholesterol compared to mice fed with standard chow, no matter in male or female mice $(24,25,26,27)$. In this regard, a high-fat diet is sufficient to establish the model of insulin resistance in the present study. GO and KEGG analyses of dysregulated genes showed that the immune response, the inflammatory response and

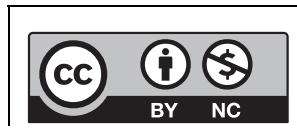

This work is licensed under a Creative Commons Attribution-NonCommercial 4.0 International License. 
metabolic pathways were mainly affected by sustained estrogen stimulation and high-fat diet. Moreover, IPA analysis showed that common, differentially expressed genes are enriched in more specific canonical pathways. Among the enriched pathways, acute phase response signaling, dendritic cell maturation and NF-кB signaling were activated, while PPAR signaling and the LXR/RXR activation pathway were markedly inhibited. These findings are also supported by recent cancer research results. For example, the NF- $\mathrm{kB}$ signaling pathway activation is closely related to chronic inflammation of the tumor $(28,29,30)$. Additionally, several studies have shown that there is crosstalk between the PPAR gamma and Wnt/beta catenin pathway systems, with them working oppositely in some diseases involved in metabolic syndromes, cancers and Alzheimer Disease (31, 32). PPARs also regulate the expression of genes involved in energy homeostasis, cell proliferation and apoptosis $(33,34)$. Furthermore, PPARs can form heterodimers with retinoid $\mathrm{X}$ receptor (RXR) modulating the expression of genes involved in lipid metabolism, adipogenesis, the maintenance of metabolic homeostasis and inflammation, as well as inducing anticancer effects in a variety of human tumors (35). Therefore, estrogen and insulin resistance may play crucial roles in tumor formation by inhibiting PPAR signaling and the LXR/RXR activation pathway in the early stages of endometrial disease.

Crosstalk between estrogen and insulin resistance is essential for neoplastic transformation in endometrial tissues (36). These two factors are likely to play collaborative roles in endometrial tumorigenesis. Interestingly, among the genes of intersection between E2 + HFD and E2 group or between E2+HFD and HFD group, we found that highfat diet improves one-third of estrogen-regulated genes and estrogen enhances one-half of high-fat diet-regulated genes. However, the antagonistic effect of estrogen and insulin resistance was weak. This suggested that the synergistic effect of these two factors is important for the initiation of endometrial lesions.

TCGA database of UCEC has facilitated the analysis of the high-throughput data of various genomic alterations. The degree of aberrant gene expression in patients emphasized the high incidence of differential genes in EC patients and helped to screen for possible diagnostic markers for early endometrial lesions. This included PIGR, KCTD1, ARG2, PMAIP1, ALPL and SLC27A2, FMO2 and CCND2. For example, SLC27A2, as the fatty acid transporter and cholesterol homeostasis mediator, was increased after E2 and/or HFD treatment, and it was also remarkably higher in the UCEC than in the para-tumorous uterine tissues. Hansel D and coworkers showed that SLC27A2 is positively involved in tumor metastasis in well-differentiated pancreatic endocrine neoplasms (37). Therefore, it could be suspected that a high level of several fatty acids may promote initiation and progression of EC.

In summary, the spectrum of crosstalk between sustained estrogen exposure and insulin resistance can be observed in our ovariectomized C57BL/6 mouse model. One way that this crosstalk occurs is through enhancing reciprocal signal activity by influencing the immune response, metabolic processes or shaping the inflammatory microenvironment of the endometrium. Thus, crosstalk between estrogen and insulin resistance, especially transcriptional enhancement, represents a potential therapeutic target for EC. Further investigation into this possibility is warranted.

\section{Supplementary data}

This is linked to the online version of the paper at https://doi.org/10.1530/ EC-17-0315

\section{Declaration of interest}

The authors declare that there is no conflict of interest that could be perceived as prejudicing the impartiality of the research reported.

\section{Funding}

This work was supported by National Natural Science Foundation of China (grant numbers 81370688, 81671417).

\section{Acknowledgements}

The authors thank the GMINIX Company (Shanghai, China) for their help in chip procedures and microarray data analysis and Dr Qizhi He for kind pathological instruction for our work.

\section{References}

1 Siegel RL, Miller KD \& Jemal A. Cancer statistics, 2017. CA: A Cancer Journal for Clinicians 201767 7-30. (https://doi.org/10.3322/ caac.21387)

2 Kaaks R, Lukanova A \& Kurzer MS. Obesity, endogenous hormones, and endometrial cancer risk: a synthetic review. Cancer Epidemiology, Biomarkers and Prevention 200211 1531-1543.

3 Key TJ \& Pike MC. The dose-effect relationship between 'unopposed' oestrogens and endometrial mitotic rate: its central role in explaining and predicting endometrial cancer risk. British Journal of Cancer 1988 57 205-212. (https://doi.org/10.1038/bjc.1988.44)

4 Berstein LM, Kvatchevskaya JO, Poroshina TE, Kovalenko IG, Tsyrlina EV, Zimarina TS, Ourmantcheeva AF, Ashrafian L \& Thijssen JH. Insulin resistance, its consequences for the clinical course of the disease, and possibilities of correction in endometrial cancer. Journal of Cancer Research and Clinical Oncology 2004130 687-693. (https://doi.org/10.1007/s00432-004-0587-2)

5 Burzawa JK, Schmeler KM, Soliman PT, Meyer LA, Bevers MW, Pustilnik TL, Anderson ML, Ramondetta LM, Tortolero-Luna G,

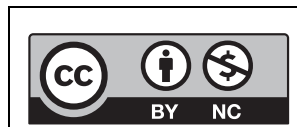

This work is licensed under a Creative Commons Attribution-NonCommercial 4.0 International License. 
Urbauer DL, et al. Prospective evaluation of insulin resistance among endometrial cancer patients. American Journal of Obstetrics and Gynecology 2011204 355.e1-357.e1. (https://doi.org/10.1016/j. ajog.2010.11.033)

$6 \mathrm{Mu} \mathrm{N}$, Zhu Y, Wang Y, Zhang H \& Xue F. Insulin resistance: a significant risk factor of endometrial cancer. Gynecologic Oncology 2012125 751-757. (https://doi.org/10.1016/j.ygyno.2012.03.032)

7 Shan W, Ning C, Luo X, Zhou Q, Gu C, Zhang Z \& Chen X. Hyperinsulinemia is associated with endometrial hyperplasia and disordered proliferative endometrium: a prospective cross-sectional study. Gynecologic Oncology 2014132 606-610. (https://doi. org/10.1016/j.ygyno.2014.01.004)

8 Calle EE \& Kaaks R. Overweight, obesity and cancer: epidemiological evidence and proposed mechanisms. Nature Reviews Cancer 20044 579-591. (https://doi.org/10.1038/nrc1408)

9 Che Q, Liu BY, Liao Y, Zhang HJ, Yang TT, He YY, Xia YH, Lu W, He XY, Chen Z, et al. Activation of a positive feedback loop involving IL-6 and aromatase promotes intratumoral 17betaestradiol biosynthesis in endometrial carcinoma microenvironment. International Journal of Cancer 2014135 282-294. (https://doi. org/10.1002/ijc.28679)

10 Barry JA, Azizia MM \& Hardiman PJ. Risk of endometrial, ovarian and breast cancer in women with polycystic ovary syndrome: a systematic review and meta-analysis. Human Reproduction Update 201420 748-758. (https://doi.org/10.1093/humupd/dmu012)

11 Ning C, Xie B, Zhang L, Li C, Shan W, Yang B, Luo X, Gu C, He Q, Jin $\mathrm{H}$, et al. Infiltrating macrophages induce ERalpha expression through an IL17A-mediated epigenetic mechanism to sensitize endometrial cancer cells to estrogen. Cancer Research 201676 1354-1366. (https://doi.org/10.1158/0008-5472.CAN-15-1260)

12 De Marco P, Bartella V, Vivacqua A, Lappano R, Santolla MF, Morcavallo A, Pezzi V, Belfiore A \& Maggiolini M. Insulin-like growth factor-I regulates GPER expression and function in cancer cells. Oncogene 201332 678-688. (https://doi.org/10.1038/onc.2012.97)

13 Lv QY, Xie BY, Yang BY, Ning CC, Shan WW, Gu C, Luo XZ, Chen XJ, Zhang ZB \& Feng YJ. Increased TET1 expression in inflammatory microenvironment of hyperinsulinemia enhances the response of endometrial cancer to estrogen by epigenetic modulation of GPER. Journal of Cancer 20178 894-902. (https://doi.org/10.7150/jca.17064)

14 MacNeil AJ, Jiao SC, McEachern LA, Yang YJ, Dennis A, Yu H, Xu Z, Marshall JS \& Lin TJ. MAPK kinase 3 is a tumor suppressor with reduced copy number in breast cancer. Cancer Research $2014 \mathbf{7 4}$ 162-172. (https://doi.org/10.1158/0008-5472.CAN-13-1310)

15 Kandoth C, Schultz N, Cherniack AD, Akbani R, Liu Y, Shen H, Robertson AG, Pashtan I, Shen R, Benz CC, et al. Integrated genomic characterization of endometrial carcinoma. Nature 2013497 67-73. (https://doi.org/10.1038/nature12113)

16 Frasor J, Weaver A, Pradhan M, Dai Y, Miller LD, Lin CY \& Stanculescu A. Positive cross-talk between estrogen receptor and NF-kappaB in breast cancer. Cancer Research 200969 8918-8925. (https://doi.org/10.1158/0008-5472.CAN-09-2608)

17 Tang L, Wang TT, Wu YT, Zhou CY \& Huang HF. High expression levels of cyclin B1 and Polo-like kinase 1 in ectopic endometrial cells associated with abnormal cell cycle regulation of endometriosis. Fertility and Sterility 200991 979-987. (https://doi.org/10.1016/j. fertnstert.2008.01.041)

18 Saltzman BS, Doherty JA, Hill DA, Beresford SA, Voigt LF, Chen C $\&$ Weiss NS. Diabetes and endometrial cancer: an evaluation of the modifying effects of other known risk factors. American Journal of Epidemiology 2008167 607-614. (https://doi.org/10.1093/aje/ kwm333)

19 Dossus L, Lukanova A, Rinaldi S, Allen N, Cust AE, Becker S, Tjonneland A, Hansen L, Overvad K, Chabbert-Buffet N, et al. Hormonal, metabolic, and inflammatory profiles and endometrial cancer risk within the EPIC cohort - a factor analysis. American Journal of Epidemiology 2013177 787-799. (https://doi.org/10.1093/ aje/kws309)

20 Yang $\mathrm{CH}$, Almomen A, Wee YS, Jarboe EA, Peterson CM \& JanátAmsbury MM. An estrogen-induced endometrial hyperplasia mouse model recapitulating human disease progression and genetic aberrations. Cancer Medicine 20154 1039-1050. (https://doi. org/10.1002/cam4.445)

21 Sah SP, Singh B, Choudhary S \& Kumar A. Animal models of insulin resistance: a review. Pharmacological Reports 201668 1165-1177. (https://doi.org/10.1016/j.pharep.2016.07.010)

22 Samuel VT, Petersen KF \& Shulman GI. Lipid-induced insulin resistance: unravelling the mechanism. Lancet 2010375 2267-2277. (https://doi.org/10.1016/S0140-6736(10)60408-4)

23 Tamemoto H, Kadowaki T, Tobe K, Yagi T, Sakura H, Hayakawa T, Terauchi Y, Ueki K, Kaburagi Y, Satoh S, et al. Insulin resistance and growth retardation in mice lacking insulin receptor substrate-1. Nature 1994372 182-186. (https://doi.org/10.1038/372182a0)

24 Fraulob JC, Ogg-Diamantino R, Fernandes-Santos C, Aguila MB \& Mandarim-de-Lacerda CA. A mouse model of metabolic syndrome: insulin resistance, fatty liver and non-alcoholic fatty pancreas disease (NAFPD) in C57BL/6 mice fed a high fat diet. Journal of Clinical Biochemistry and Nutrition 201046 212-223. (https://doi.org/10.3164/ jcbn.09-83)

25 Do GM, Oh HY, Kwon EY, Cho YY, Shin SK, Park HJ, Jeon SM, Kim E, Hur CG, Park TS, et al. Long-term adaptation of global transcription and metabolism in the liver of high-fat dietfed C57BL/6J mice. Molecular Nutrition and Food Research 2011 55 (Supplement 2) S173-S185. (https://doi.org/10.1002/ mnfr.201100064)

26 Masi LN, Martins AR, Rosa Neto JC, do Amaral CL, Crisma AR, Vinolo MA, de Lima Júnior EA, Hirabara SM \& Curi R. Sunflower oil supplementation has proinflammatory effects and does not reverse insulin resistance in obesity induced by high-fat diet in C57BL/6 mice. Journal of Biomedicine and Biotechnology 20122012945131. (https://doi.org/10.1155/2012/945131)

27 Wong SK, Chin K-Y, Hj Suhaimi F, Fairus A \& Ima-Nirwana S. Animal models of metabolic syndrome: a review. Nutrition and Metabolism 201613 65. (https://doi.org/10.1186/s12986-016-0123-9)

28 Che Q, Liu B-Y, Wang F-Y, He Y-Y, Lu W, Liao Y, Gu W \& Wan XP. Interleukin 6 promotes endometrial cancer growth through an autocrine feedback loop involving ERK-NF-kappaB signaling pathway. Biochemical and Biophysical Research Communications 2014 446 167-172. (https://doi.org/10.1 016/j.bbrc.2014.02.080)

29 Huang Y, Chen R \& Zhou J. E2F1 and NF-kappaB: key mediators of inflammation-associated cancers and potential therapeutic targets. Current Cancer Drug Targets 201616 765-772. (https://doi.org/10.217 4/1568009616666160216130755)

30 Zhang J, Song H, Lu Y, Chen H, Jiang S \& Li L. Effects of estradiol on VEGF and bFGF by Akt in endometrial cancer cells are mediated through the NF-kappaB pathway. Oncology Reports 201636 705-714. (https://doi.org/10.3892/or.2016.4888)

31 Lecarpentier Y, Claes V, Vallée A \& Hébert J-L. Interactions between PPAR gamma and the canonical Wnt/Beta-catenin pathway in type 2 diabetes and colon cancer. PPAR Research 201720175879090. (https://doi.org/10.1155/2017/5879090)

32 Vallee A \& Lecarpentier Y. Alzheimer disease: crosstalk between the canonical Wnt/Beta-Catenin pathway and PPARs alpha and gamma. Frontiers in Neuroscience 201610 459. (https://doi.org/10.3389/ fnins.2016.00459)

33 Ahmadian M, Suh JM, Hah N, Liddle C, Atkins AR, Downes M \& Evans RM. PPARgamma signaling and metabolism: the good, the bad and the future. Nature Medicine 201319 557-566. (https://doi. org/10.1038/nm.3159) http://www.endocrineconnections.org

https://doi.org/10.1530/EC-17-0315
() 2018 The authors

Published by Bioscientifica Ltd

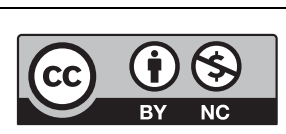

This work is licensed under a Creative Commons Attribution-NonCommercial 4.0 International License. 
34 Vitale SG, Laganà AS, Nigro A, La Rosa VL, Rossetti P, Rapisarda AM, La Vignera S, Condorelli RA, Corrado F, Buscema M, et al. Peroxisome proliferator-activated receptor modulation during metabolic diseases and cancers: master and minions. PPAR Research 201620166517313. (https://doi.org/10.1155/2016/6517313)

35 Fanale D, Amodeo V \& Caruso S. The interplay between metabolism, PPAR signaling pathway, and cancer. PPAR Research 20172017 1830626. (https://doi.org/10.1155/2017/1830626)

36 Zhang Q, Celestino J, Schmandt R, McCampbell AS, Urbauer DL, Meyer LA, Burzawa JK, Huang M, Yates MS, Iglesias D, et al.
Chemopreventive effects of metformin on obesity-associated endometrial proliferation. American Journal of Obstetrics and Gynecology 2013209 24.e1-24.e12. (https://doi.org/10.1016/j. ajog.2013.03.008)

37 Hansel DE, Rahman A, House M, Ashfaq R, Berg K, Yeo CJ \& Maitra A. Met proto-oncogene and insulin-like growth factor binding protein 3 overexpression correlates with metastatic ability in well-differentiated pancreatic endocrine neoplasms. Clinical Cancer Research 200410 6152-6158. (https://doi.org/10.1158/1078-0432. CCR-04-0285)

Received in final form 8 November 2017

Accepted 10 November 2017

Accepted preprint published online 13 November 2017 http://www.endocrineconnections.org https://doi.org/10.1530/EC-17-0315
() 2018 The authors Published by Bioscientifica Ltd

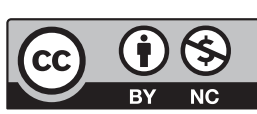

This work is licensed under a Creative Commons Attribution-NonCommercial 4.0 International License. 